\title{
Laryngeal Nerves Monitoring Versus Non-monitoring in Thyroid Surgery
}

\author{
Mohammed Fatehy Zidan, Ahmed Abdou Moustafa
}

Departments of ENT and Surgery Faculty of Medicine for Girls, Al-Azhar University

\begin{abstract}
Background and objectives: Thyroid surgery is the most common cause of recurrent nerve (RLN) injury. Deliberate identification of the RLN minimizes the risk of injury. When the nerve is identified and dissected, the reported RLN injury rate during thyroidectomy is $0-2.1 \%$. Continuous intra-operative nerve monitoring during surgery remains a controversial issue. The basic technique involves a skin surface electrode or muscle electrode used to make electromyography (EMG) recordings, which have an audible alarm to alert the surgeon if passive (e.g.stretch during traction) or active nerve stimulation has occurred. We aim to evaluate the use of intra-operative nerve monitoring (IONM) to preserve the laryngeal nerves that may be at risk for injury during thyroid surgery and show the merits of using electrophysiologic laryngeal nerve monitoring during thyroid surgery.
\end{abstract}

Patients and methods: This study was conducted as a prospective study on 28 patients whom are thyroidectomy candidates; we elected 14 thyroidectomy candidates in whom we don't use NIM during surgery (Control group_A). These patients were chosen to be evenly matched with another 14 thyroidectomy candidates to monitor the recurrent laryngeal (RLN) and external branch of superior laryngeal (EBSLN) throughout thyroidectomy procedures (NIM group_ B) to compare the laryngeal nerves risk of injury with and without the use of nerve monitor.

Results: Four patients ( 2 patients in-group A and 2 patients in-group B) were dysphonic after operation. Laryngoscopy revealed unilateral recurrent laryngeal nerve palsy in all except one bilateral in group A. There were no significant differences in RLN paralysis, paresis, or total injury rates between both groups. The number of patients in the presented study was limited to draw a statistical conclusion for significance.

Conclusions: The routine application of IONM cannot prevent or reduce recurrent laryngeal nerve injury. However the study signifies the trend towards the use of INOM in expectedly difficult cases and in revision surgery with distorted anatomical relationships and fibrous adhesions.

\section{Introduction}

Thyroid surgery is the most common cause of recurrent nerve (RLN) injury, and gives a rate approaching 5\% in a large series with close follow-up (Lo C et al; 2000). These complications are probably more common in revision neck surgery.

The course of the RLN frequently varies despite normal anatomy or as consequence of congenital vascular anomalies or distortion of regional anatomy by extension of goiter by neoplasm or inflammation (Henry et al., 1988). When approaching the inferior pole of the gland, the RLN may be traversed by the ITA (inferior thyroid artery) or may pass between the arterial ramifications.

Deliberate identification of the RLN minimizes the risk of injury. When the nerve is identified and dissected, the reported RLN injury rate during thyroidectomy is $0-2.1 \%$. This rate is reportedly higher if surgery is repeated (2$12 \%)$ or if the nerve is not clearly identified (4-6.6\%). Intraoperative hemostasis and a thorough understanding of the anatomy are essential for identifying and preserving the nerve. Even the most experienced surgeon may have difficulty identifying and preserving cranial nerves. Intra-operative nerve monitoring is an important adjunct to 
enhance nerve preservation, particularly when tumor, infection, trauma or anatomic variations place the nerve at an increased risk (Kartush and Bouchard, 1992).

An extremely important variation of the pathway of the RNL depends on congenital vascular alterations involving the major vessels at the root of the neck. Rarely $(0.6 \%)$, the right recurrent laryngeal nerve passes directly from the vagus in the neck towards the larynx as a "non-recurrent" inferior laryngeal nerve that makes it highly susceptible to surgical injury (Ardito et al., 1998). The possibility of non-recurrence of the inferior laryngeal nerve on the left side is a very rare exception and it is reported in an autopsy case (Berlin, 1935) and in two clinical cases (Henry, 1988) associated with a right-sided aorta and a left retroesophageal subclavian artery.

There is an inverse relationship between the number of thyroid surgeries performed and complication rate. Permanent nerve palsy is cited in the literature to occur in $0 \%$ to $2.1 \%$, with an average of approximately $0.5 \%$ to $1 \%$. Temporary palsy varies from $2.9 \%$ to over $10 \%$. The right-sided nerve is at higher risk due to its wide anatomical variation as compared to the left one.

The aim of this article is to show how to preserve the important neck nerves during Thyroid surgery. Also, evaluate the use of intra-operative nerve monitoring to preserve the laryngeal nerves that may be at risk for injury during thyroid surgery. Also we tried to know the merits of using electrophysiologic laryngeal nerve monitoring during thyroid surgery.

\section{Patients and Methods:}

Patients who had been Thyroidectomy candidates were subjected to appropriate Pre-operative assessment. Case history, General and Local examination of the neck, Thyroid function test, ultrasound of the neck, Thyroid scan using radioactive iodine uptake, FNA, CT scan, Indirect laryngoscopy or flexible nasopharyngoscopy for vocal fold mobility and Subjective voice assessment.
We described 14 cases (group B) of thyroidectomy candidates in which we elected to monitor the recurrent laryngeal (RLN) and external branch of superior laryngeal (EBSLN). These patients were chosen to be evenly matched with another 14 patients as control (group A) regarding distribution of pathology and anticipated difficulty of surgery; in which RLNs are not monitored during thyroidectomy. We excluded from this study Patients involved with neoplasm and Patients with preoperative RLNs injuries. In this study we included 14 cases of multinodular goiter, 9 cases of thyroiditis uncontrolled with medical treatment and 5 cases of solitary thyroid nodule.

All patients had total thyroidectomy, bringing the total number of RLNs dissected to 56 nerves. Twenty eight consecutive RLNs (group A) were unmonitored to be identified anatomically and surgically, and the other twenty eight consecutive RLNs (group B) were monitored intraoperative throughout surgical manipulations. General anesthesia was administered; however, long acting muscle relaxants were avoided in monitored group.

\section{Exposure of RLN:}

In all cases we identified RLNs by using a low anterior approach. In this approach, the strap muscles are separated in the midline down to the sternal notch. The strap muscles are reflected laterally, and dissection is carried out in the paratracheal regions. The right or left RLN is identified in the fat and nodal tissue and dissected superiorly to allow visual confirmation of its location during the surgery.

Early in the operation the RLN is identified in the inferior pole area and traced to the cricothyroid membrane, avoiding its injury by keeping it in direct view during cauterization and division of the tissues. Also, the RLN can be identified as it enters the larynx with dissection in the vascular space between the superior thyroid pole and the larynx. This can be done without taking down the superior pole vessels by gently retracting the superior pole laterally and dissecting along the larynx to the laryngotracheal groove, where the RLN 
frequently branches before disappearing under the cricopharyngeus. The RLN can be traced inferiorly and identified in the hilum of the thyroid and further inferiorly, if necessary. If the nerve was not located in its expected location, dissection and stimulation to detect an aberrant or nonRLN is recommended.

In monitored group Recording electrodes were positioned to monitor both the right and left vocal folds. An identical fine silver wire electrode was used, applied to the vocal folds. We used a laryngoscope to obtain visualization of the vocal folds and placed the needle into the vocalis musculature with a long alligator forceps. A ground electrode for the laryngeal surface electrode was placed on the patient forehead or beneath the clavicle. The RLN was stimulated by the application of a unipolar brass probe to deliver an electric current that ranged from 0.5 to $1.5 \mathrm{~mA}$ at a frequency of $30 \mathrm{~Hz}$. The identity of an intact RLN would be confirmed through a series of audible acoustic signals that were generated by the machine and an action potential traced on a monitor screen. The functional integrity of the nerve once again should be confirmed at the end of the thyroidectomy by the testing of the most proximal exposed portion of the nerve. The absence of a signal that was generated by the stimulator at any precise point along the nerve would be regarded as a positive test for nerve compromise. Postoperatively, patient voice was assessed by the surgeon by listening to the patient's voice and asking the patient about vocal quality. Also, vocal fold examination was performed for all patients postoperatively within 2 weeks after the surgical procedure using an indirect or flexible laryngoscope.

Any reduction in the movement of the vocal fold was recorded as postoperative vocal fold paralysis. For those patients, repeated examinations were performed periodically at $1,3,6$, and 12 months after the operation until full recovery of vocal fold function had been confirmed. The presence of vocal fold paralysis for more than 12 months after thyroidectomy was regarded as permanent paralysis.

\section{Fig (1): Recurrent laryngeal nerve Identification during thyroid surgery}

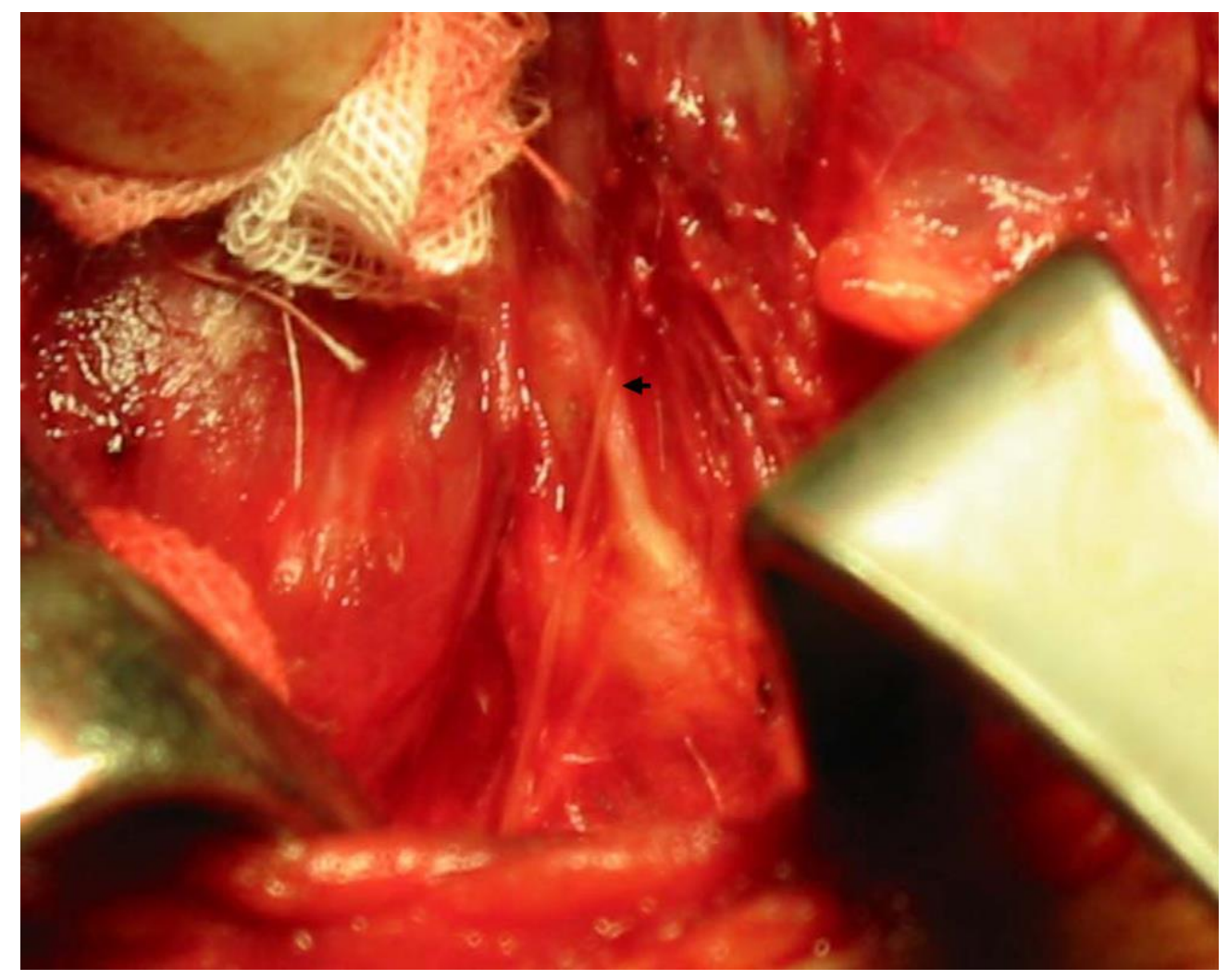




\section{Exposure of EBSLN:}

Visualization of the ESLN is not routinely obtained during thyroidectomy, because of the small diameter of this branch $(0.2 \mathrm{~mm})$, its very variable course, and the anatomic position (the space between the medial surface of the upper pole of the thyroid and the cricothyroid muscle, which is covered by the strap muscles). In about $10 \%$ to $15 \%$ of cases, the nerve runs within the cricothyroid muscle in its entire course, so it cannot be seen by direct inspection using any technique, but, in this case it is not at risk. So, thyroidectomy was performed either with identification of the EBSLN before ligation of the superior thyroid pole or thyroidectomy included skeletonization and individual ligation of the superior pole vessels adjacent to the capsule of the gland. The superior pole dissection and ligation of the superior thyroid artery and veins are the initial steps of our surgical procedure. The vessels are exposed at their penetration point of the thyroid capsule in this area. EBSLN may be closely related to the superior thyroid vessels, usually medial. We separate the arterial and venous branches of the upper thyroid pole in several small steps near to the thyroid capsule.

Also, one of the common techniques which used for ligating the superior thyroid pedicle is to dissect from medial to lateral with a right angle clamp after creating the medial thyroid space and ligating the vessels individually close to the thyroid. Three additional maneuvers needed are as follows: (1) the upper pole should be lateralized, (2) direct visualization of the EBSLN should be attempted on the medial side of the pedicle and, (3) superior thyroid vessels should be individually ligated. This technique defined as "lateralization of superior pole."

For neuron-monitored cases of EBSLN, the electrode is placed into the cricothyroid muscle, and the nerve is stimulated by direct attachment with the stimulation probe $(0.5$ to $1.0 \mathrm{~mA}, 30 \mathrm{~Hz})$. The positive result of this stimulation is heard from an audible signal and also is seen as a corresponding contraction of the cricothyroid muscle.

By using the neuromonitoring procedure we can exclude the presence of EBSLN in each of these portions step by step before dissection and ligation. A present EBSLN or branch of it will be isolated carefully. In this manner the upper pole can be mobilized completely without damaging the EBSLN. The course can be demonstrated, and the intact function of the EBSLN can be checked then easily by neurostimulation of the nerve.

Because all intraoperative monitoring requires that EMG activity be recorded from a muscle or muscles, it is important that the muscles not be paralyzed by using of paralyzing anesthetic agents during the surgery or at least during those parts of the surgery when the nerve being monitored is deemed at risk by the surgeon. Short acting neuromuscular depolarizing agents could be used at the induction of anesthesia for endotracheal intubation.

When the case is complete and before closing the surgical site, the surgeon should evaluate the nerves functional integrity one more time. This should be done by stimulating the nerve both proximal and distal to the immediate dissection area as far as is accessible within the surgical field. Use the lowest level of stimulation that produces a response. We continue to monitor with the NIM (Nerve integrity monitor)-Response system throughout the procedure (until the incision is closed), stress or pressure such as wound dressing might exert stress or pressure on the nerve and affect its function.

\section{Results}

Twenty eight patients were randomly assigned into group A (control group) and B Intra-operative neuromonitoring (IONM group); 14 patients each. The two groups were matched as regards to age, sex, type of operation, and thyroid pathological findings. 
There were 9 women and 5 men with range of age (15-81 years) in group A, 8 women and 6men with range of age (2085 years) in group B. All patients underwent total thyroidectomy. Clinical examination revealed toxic and simple nodular goiter and thyroiditis. The operative time was higher in-group A about 120 minutes, than in group B which was about 100 minutes. Results were evaluated based on the postoperative indirect laryngoscopy or flexible nasopharyngoscopy.
Three patients (2 patients in-group A and 1 patient in-group $B$ ) were dysphonic after operation. Laryngoscopy revealed unilateral recurrent laryngeal nerve palsy in one in group B while one in group A was unilateral and the second was bilateral. In these cases, it was not possible to evaluate whether an EBSLN injury was present. In all patients, the symptoms spontaneously disappeared after 6 months, except one.

In the patient of group A with bilateral vocal cord postoperatively, he had only sluggish mobility of right(rt) vocal cord while the left(lt) cord was completely immobile, however rt cord completely recover mobility before six months with only partial recovery of the lt cord at the sixth month causing light dysphonia without airway compromise. This patient suffered huge goiter long time before being operated (12 years), his goiter was extending deeply retrosternal which entailed prolonged manipulation and traction to deliver. He had been suffering recurrent goiter symptoms for over 12 years, having had a subtotal thyroidectomy23 years ago for benign multi-nodular disease. Preoperatively, the patient suffered difficult breathing and positive Pemberton's sign, CT images showed the presence of a huge retrosternal goiter with tracheal deviation extending above the aortic arch and Superior cava vein was displaced anteriorly. When a goiter is purely cervical; however, it rarely compresses the trachea to cause obstruction. On surgery the trachea was normal with no features of tracheomalacia. The surgeon opens the interclavicular ligament, finds the correct plane for careful digital mediastinal exploration, and carefully brings the retrosternal part of the gland all the way through the thoracic inlet into the cervical incision. In this case, this step was possible with digital dissection; the whole mass was then enucleated. A tracheostomy was placed prophylactically at thyroidectomy completion and had been removed on $10^{\text {th }}$ day post-operatively.

Another patient in group B (IONM) showed similar findings of huge retrosternal goiter, however a subcapsular identification of the RLN could be made with the stimulator probe, and the nerve had been peeled off the gland prior to delivery into the cervical field. Anytime the recurrent laryngeal nerve was stretched by the traction during delivery into the cervical field, the electrical discharge on the recording alerted us of RLN compromise. However, Total thyroidectomy was uneventful in this patient with no complications.

The results of phoniatric evaluation performed 6 months after the operation to evaluate EBSLN in all patients with transient inferior laryngeal nerve injury were normal. One patient in-group A presented with phonasthenia and decreased range of pitch volume after operation without evidence of alterations during indirect laryngoscopy. In this patient, the symptoms spontaneously disappeared after 2 months.
In patients who presented with no symptoms after operation, indirect laryngoscopy showed the absence of inferior displacement of the vocal fold, bowing of the vocal fold, nor ipsilateral posterior glottic rotation, in both groups of patients. No voice changes were documented on the second postoperative day or 1 and 6 months after surgery.

In-group B, ten cases were depicted accurately by the neuromonitoring (true positive), however in two patients, the RLN was visually identified during the 
surgery but this finding was not supported by an electromyographic response with the nerve stimulator. In these cases, the whole nerve monitoring system was checked; the needle was then checked and replaced into the vocalis musculature correctly for the proper setup and was found to function in these two cases. However, intact

Fig (2): (A \& B) Normal vocal folds,
(A) Respiration
(B) Phonation
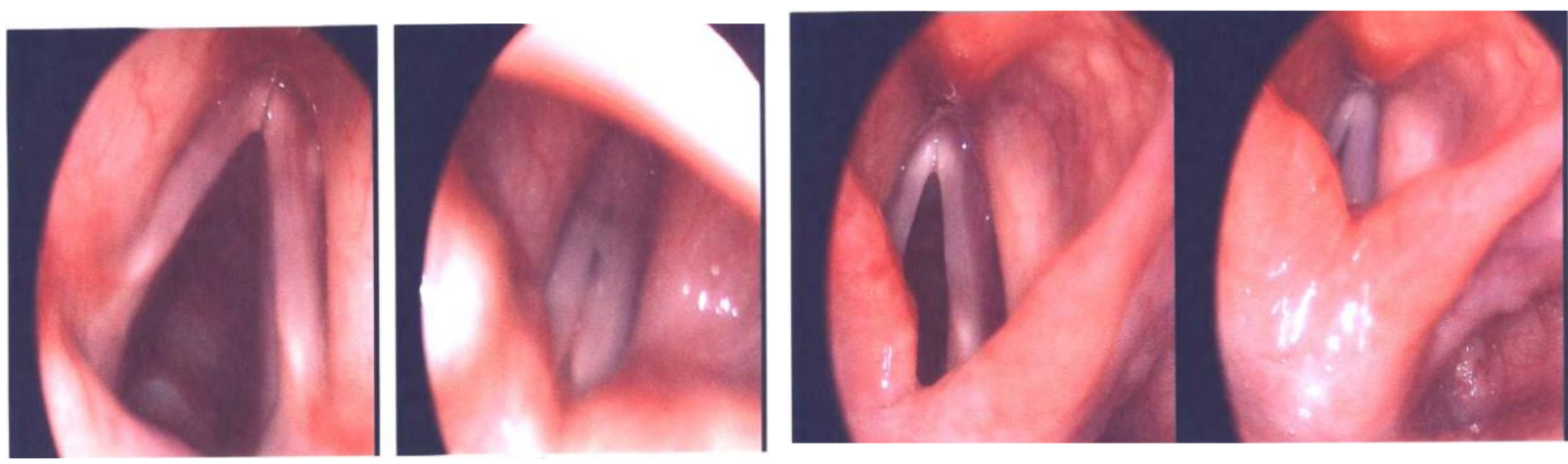

Summery of Results

\begin{tabular}{|l|l|l|}
\hline & Group A (Control group) & $\begin{array}{l}\text { Group B (IONM group) } \\
\text { (Intra-operative neuromonitoring) }\end{array}$ \\
\hline No. of cases & $14(26$ nerves) & $14(26$ nerves) \\
\hline Age & $15-81$ & $20-85$ \\
\hline $\begin{array}{l}\text { Sex } \\
\text { F }\end{array}$ & 5 & 6 \\
\hline Intervention & 9 & 8 \\
\hline Operative time & Total thyroidectomy & Total thyroidectomy \\
\hline $\begin{array}{l}\text { No of RLN paresis } \\
\text { (Temporarily on immediate postop. exam) }\end{array}$ & 120 min & $\mathbf{1 0 0}$ min \\
\hline $\begin{array}{l}\text { No of EBSLN paresis } \\
\text { (Temporarily on immediate postop. exam) }\end{array}$ & 1 & 1 \\
\hline No of persistent palsy & $1 *$ Patients; 1bilat. 1unilat.) & $\mathbf{0}$ \\
\hline Postoperative hospital stay & $3-4$ d & $\mathbf{0}$ \\
\hline $\begin{array}{l}\text { Pathology } \\
\text { Multinodular Goiter } \\
\text { Toxic Thyroiditis } \\
\text { Solitary Benign Nodule }\end{array}$ & Benign & $3-4$ d \\
\hline Retro-Sternal Goiter (RSG) & $\mathbf{2}$ & $\mathbf{B e n i g n}$ \\
\hline
\end{tabular}

*(patient with persistent dysphonia and impaired left cord mobility after 6 months) 


\section{Discussion:}

Continuous intra-operative nerve monitoring during surgery remains a controversial issue. The basic technique involves a skin surface electrode used to make electromyography (EMG) recordings, which have an audible alarm to alert the surgeon if passive or active nerve stimulation has occurred. It is relatively easy to use and interpret and although rare complications including skin burns secondary to technical defects have been reported, it is relatively safe and reliable (Haenggeli et al., 2000).

Our study used the Medtronic Xomed NIM (Nerve integrity monitor) - response (4channels). This technique allows for monitoring of motor nerves of the neck as it senses a change in the baseline electrical activity of their musculature. The number of motor nerve injures of the neck were compared with and without use of electrophysiological monitoring during Thyroidectomy surgeries. This study was a multi-institutional prospective study with 3 years follow up for the cases. Two centers of them did not use the Intra-operative nerve monitoring (IONM) at all and one center used IONM in all surgeries. In addition, this study was done between ENT department and general surgery department in Al-Zahra hospital.

In the present study there was no statistically significant difference in nerve paralysis, paresis, or total nerve injury numbers between control and NIM groups; however the incidence of postoperative nerve paresis was higher in control group, which was compatible with several other studies.

Intra-operative nerve monitoring (IONM) is by no means a substitute for careful and appropriate dissection during surgery. Benefits of intra-operative nerve monitoring may include, immediate feedback of mechanically evoked potentials to prompt the surgeon to modify the technique or location of the dissection, verification of nerve location once identified, verification of nerve integrity at the end of the procedure to help in decision making (i.e., the extent of dissection on the contra-lateral side) as well as a guide when anatomy is distorted by malignancy or reoperation (Hermann et al., 2004).

To date, no controlled prospective trials show that there is a reduced rate of nerve injury with intraoperative nerve monitoring. This was, in fact, a reason cited by several surgeons for not using nerve monitoring (Horne et al., 2007). Most current approaches to thyroid surgery emphasize preservation of the RLN, with recent studies advocating the importance of intraoperative nerve identification and protection rather than avoidance (Affleck et al., 2003). The most striking evidence to support this is a review by (Hermann et al., 2002) of 16,443 patients undergoing thyroidectomy. With nerve exposure ranging from mere identification to complete exposure, there was a lower RLN injury rate with increasing level of dissection.

In a multi-institutional prospective study, Dralle et al., 2004 examined almost 30,000 RLNs at risk during thyroid surgery and found no significant difference in the rate of injury to the RLN when comparing intraoperative dissection identification vs. identification with continuous nerve monitoring. A recent retrospective review by (Witt, 2005) was performed to compare rates of RLN injury with and without the use of an RLN monitor. Of 136 cases reviewed, monitored RLNs had a temporary injury rate of 2.8 percent and a permanent injury rate of 0.9 percent. In unmonitored RLNs, these rates were 4.8 percent and 2.4 percent, respectively; neither rate reached statistical significance, but showed a clear trend.

Brennan et al., 2001 performed a prospective analysis of continuous intraoperative nerve monitoring, which showed a one percent incidence of temporary RLN injury and a zero percent incidence of permanent injury with a total of 96 nerves at risk in patients undergoing thyroid and parathyroid surgeries. While these rates of RLN injury are lower than those in most published series, it is difficult to determine whether these findings are a function of the nerve monitor or the level of experience of 
the surgeon (Horne et al., 20o7). The mechanisms of intra-operative nerve injury include division, laceration, traction, pressure, electrical insult, ligature entrapment, ischemia, and suction trauma (Brennan et al., 2001).

Stimulation threshold of the RLN at the end of the procedure were less than or equal to $0.4 \mathrm{~mA}$ in patients with normally functioning nerves post-operatively. However Stimulation thresholds greater than or equal to $0.5 \mathrm{~mA}$ may correlate with RLN paralysis postoperatively (Brennan et al., 2001).

In a large prospective multi-center trial (Thomusch et al., 2002) reported a statistical significant reduction in the permanent RLN injury rate for low- risk patients who underwent primary operations for benign goiter with the addition of electro myographic monitoring.

However ,another multi-center study that included 29,998 nerves that were at risk revealed no statistical difference in the frequency of RLN palsy with the adoption of IONM compared with the use of visual identification only. However, Surgeon`s experience combined to RLN monitoring did matter in reducing the incidence of permanent RLN palsy (Chiang et al., 2005).

In Chan et al., 2006 study, there were no statistical differences in the post-operative, transient, and permanent nerve palsy rates between the IONM and control groups. However, the incidence of postoperative RLN paresis was significantly higher in thyroidectomy for malignant disease and reoperations.

In the multivariate analysis, the use of IONM did reduce the incidence of postoperative RLN palsy for secondary thyroidectomy, thyroidectomy for malignancy and retrosternal goiter, but the sample size might be insufficient to show a statistical significant differences. In addition, when patients who underwent secondary thyroidectomy were compared, there was a positive trend for the reduction in post-operative overall RLN palsy rates from $19 \%$ to $7.8 \%$, for those procedures that were performed with IONM, while transient RLN palsy rates was reduced from $14.2 \%$ to $5.2 \%$ and permanent RLN palsy rates from $4.8 \%$ to $2.6 \%$ (Chan and Lo, 2006).

Lambert et al., 2000 said that, electrophysiologic monitoring might be more predictive if the vagus nerve is stimulated rather than the RLN. The RLN may be stimulated too close to the larynx. An injury to the RLN may be more proximal. Additionally, the loss of baseline electromyographic activity during dissection can alert the surgeon to possible nerve injury, which would prompt an investigation for potentially treatable injuries (such as a ligature or clip that could be removed). Discovery of a transected nerve could lead to primary repair in hopes of reducing long-term muscular atrophy (Yarbrough et al., 2004).

Concerns cited by many surgeons were that intraoperative RLN monitoring was the "standard of care." Those who used monitoring felt as though monitoring was, in fact, the standard of care for their community and that not monitoring could have medico-legal consequences. (Horne et al., 2007).Electro-physiologic monitoring of the RLN may then play an unintended role. Electro-physiologic monitoring was not demonstrated in this paper to reduce the incidence of transient or permanent vocal fold immobility; however, it documented the integrity of the nerve throughout the procedure.

Dackiw et al., 2002 demonstrated in their study that $(91 \%)$ of RLNs were correctly identified by the nerve monitor (truepositive cases). As noted, in 15 cases an audible stimulus was not recorded on nerve stimulation (false negative). In 10 cases, areas of (false positive) stimulation occurred. Possible explanations for the areas of false-positive and false negative stimulation include incorrect external or internal lead placement, the sensitivity of the device, and partial nerve paresis. Repositioning of the endotracheal tube electrode may correct false-negative stimulation when it occurs. Insertion of the endotracheal electrode too distally into the trachea may result in areas of false positive stimulation over the trachea. Similarly, if the sensitivity of the device is adjusted to 
be too high, areas of false-positive stimulation may occur.

Robertson et al., 2004 compared outcomes of 120 unmonitored and 116 monitored cases performed. RLN paralysis occurred in $2.5 \%$ in control group and $0.86 \%$ in the NIM group. Temporary RLN paresis occurred in $4.24 \%$ in the control group and $3.45 \%$ in the NIM group. Total RLN injury occurred in $6.8 \%$ in the control group and $4.3 \%$ in the NIM group. There were no statistical significant differences in $\mathbf{R L N}$ paralysis, paresis, or total injury rates between both groups.

The most proven method to decrease RLN injury continues to be surgical experience with a thorough knowledge of anatomy and meticulous identification and protection of the nerve along its course in the neck (Yarbrough et al., 2004). In addition, reducing RLN injury in thyroid surgery requires gentle technique, and possibly magnification.

Technology must be used with discretion in an environment of cost constraints. A procedure involving electro-physiologic monitoring requires additional time, reflecting an additional cost not only in operative time, but also in the purchase and maintenance of the nerve integrity monitor, the need for a special endotracheal tube, and the technical staff who intra-operatively monitor the RLN. Given the higher rates of permanent vocal fold immobility for patients with malignancy, substernal goiter, and revision surgery, electrophysiological monitoring is suggested for these cases (Witt, 2005).

It is important to note that, in several studies, electrophysiological RLN integrity did not always translate into clinical vocal fold mobility after surgery. This highlights the limited functional predictive value of intraoperative nerve monitoring. (Witt, 2005; Yarbrough et al.; 2004, Hermann et al.; 2004, Beldi et al., 2004)

The number of patients in the presented study was limited to draw a statistical conclusion for significance; however the study signifies the trend towards the use of INOM only in expectedly difficult cases and in revision surgery with distorted anatomical relationships and fibrous adhesions.

\section{Summary and conclusion}

In conclusion, the adoption of using IONM compared with routine nerve identification (i.e. without using IONM) in the neck surgery could not be demonstrated in our patients to reduce nerve injury significantly. The routine application of IONM cannot prevent or avoid nerve injury necessarily for all types of neck surgical procedures and can be associated with potential confusions or limitations. However, for selected high risk difficult cases like reoperations, cancer excisions, anatomic distortion with large tumors, anatomic anomalies and a history of irradiation or inflammation, the use of IONM may be associated with an improved outcome.

\section{References:}

1. Affleck, B.; Swartz, K. and Brennan, J. (2003). Surgical considerations and controversies in thyroid and parathyroid surgery. Otolaryngol Clin North Am. 36, 159187

2. Ardito, G.; Manni, R. and Vincenzoni, C. et al. (1998). II nervo laringeo inferiore non ricorrente: esperienza chirurgica. Ann Ital Chir. 69, 21-4.

3. Beldi G, Kinsbergen T, Schlumpf $\mathbf{R}$ (2004). Evaluation of intraoperative recurrent nerve monitoring in thyroid surgery. World $\mathbf{J}$ Surg.;28(6):589-91.10

4. Berlin, D.D. (1935). The recurrent laryngeal nerves in total ablation of the normal thyroid gland: an anatomical and surgical study. Surg Gynecol Obstet. 60, 19

5. Brennan, J.; Moore, E.J. and Shuller, K.J. (2001). Prospective analysis of the efficacy of continuous intra-operative nerve monitoring during thyroidectomy, parathyroidectomy and parotidectomy. Otolaryngol Head Neck Surg. 124, 537-543.

6. Chan, W.F. and Lo, C.Y. (2006). Pitfalls of intra-operative neuromonitoring in predicting postoperative recurrent laryngeal nerve function during thyroidectomy. World J Surg. 30, 806812.

7. Chan, W.F.; Lang, B.H. and Lo, C.Y. (2006). The role of intra-operative neuromonitoring of recurrent laryngeal nerve 
during thyroidectomy: A comparative study on 1000 nerves at risk. Surgery. 140, 866-873.

8. Chiang, F.Y.; Wang, L.F.; Huang, Y.F.; Lee, K.W. and Kuo, W.R. (2005). Recurrent laryngeal nerve palsy after thyroidectomy with routine identification of the recurrent laryngeal nerve. Surgery. 137, 342-347.

9. Dackiw, A.P.; Rotstein, L.E. and Clark, O.H. (2002). Computer-assisted evoked electromyography with stimulating surgical instruments for recurrent/external laryngeal nerve identification and preservation in thyroid and parathyroid operation. Surgery. 132, 11001108 .

10. Dralle, H.; Sekulla, C. and Haerting, J. (2004). Risk factors of paralysis and functional outcome after recurrent laryngeal nerve monitoring in thyroid surgery. Surgery. 136, 1310-1322.

11. Haenggeli, A.; et al. (2000). A complication of intraoperative facial nerve monitoring: facial skin burns. Am J Otol. 20, 679-82.

12. Henry, J.F.; Audiffret, J.; Denizot, A. and Plan, M. (1988). The non-recurrent inferior laryngeal nerve: review of 33 cases including two on the left side. Surgery. 104, 977-84.

13. Hermann, M.; Alk, G. and Roka, R. (2002). Laryngeal recurrent nerve injury in surgery for benign thyroid diseases: effect of nerve dissection and impact of individual surgeon in more than 27,000 nerves at risk. Ann Surg. 235, 261-268.

14. Hermann, M.; Hellebart, C. and Freissmuth, M. (2004). Neuromonitoring in thyroid surgery: prospective evaluation of intraoperative electrophysical responses for the prediction of recurrent laryngeal nerve injury. Ann Surg. 240, 9-17.

15. Horne, S.K.; Gal, T.J. and Brennan, J.A. (2007). Prevalence and patterns of intraoperative nerve monitoring for thyroidectomy. Otolaryngol Head Neck Surg. 136, 952-956.

16. Kartush, J.M. and Bouchard (1992). Neuromonitoring in Otology and Head and Neck Surgery. Raven Press. Ltd: New York(ed). 17. Lambert, A.; Cosgrove, C.; Barwell, J.; Oxenham S. and Wilkins, D. (2000). Vagus nerve stimulation: quality control in thyroid and parathyroid surgery. J Laryngol Otol. 114, 125127.

18. 7. Lo C, Kwok K, Yuen P. A (2000) .prospective evaluation of recurrent laryngeal nerve paralysis during thyroidectomy. Arch Surg 2000;135:204-7.

19. Robertson, M.L.; Steward, D.L.; Gluckman, J.L. and Welge, J. (2004). Continuous laryngeal nerve integrity monitoring during thyroidectomy: Does it reduce risk of injury? Otolaryngol Head Neck Surg. 131, 596600.

20. Thomusch, O.; Sekulla, C.; Walls, G.; Machens, A. and Dralle, H. (2002). Intraoperative neuromonitoring of surgery for benign goiter. Am J Surg. 183, 673-678.

21. Witt, R.L. (2005). Recurrent laryngeal nerve electrophysiologic monitoring in thyroid surgery: the standard of care?. J Voice. 19, 497500 .

22. Yarbrough, D.E.; Thompson, G.B.; Kasperbauer, J.L. Michel Harper, C. and Clive S.G. (2004). Intraoperative electromyographic monitoring of the recurrent laryngeal nerve in re-operative thyroid and parathyroid surgery. Surgery. 136, 1107-1115. 
دراسه مقارنه بين استخدام جهاز مر اقبة (استثعار) الاثشارت العصبيه وترك استخدامه فى جراحات استئصال الغده الدرقيه

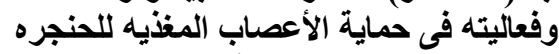

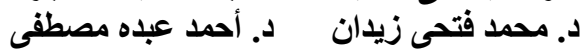

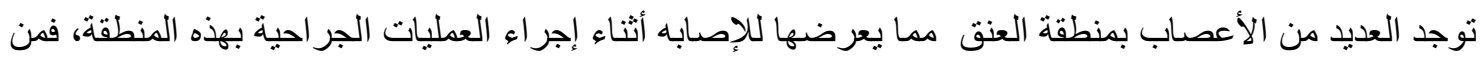

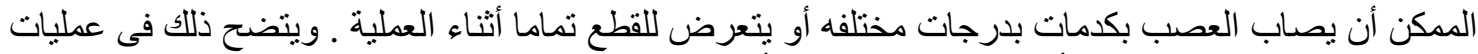

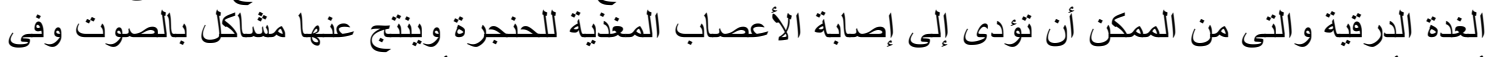

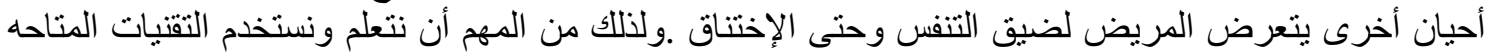

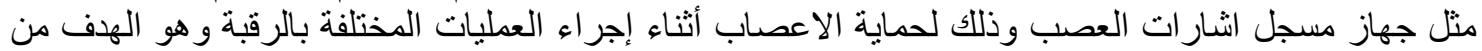

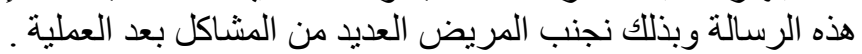

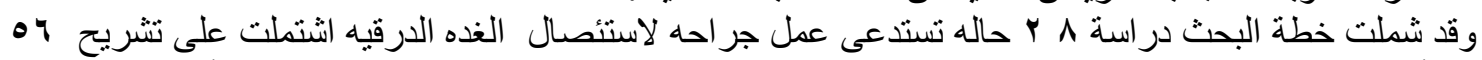

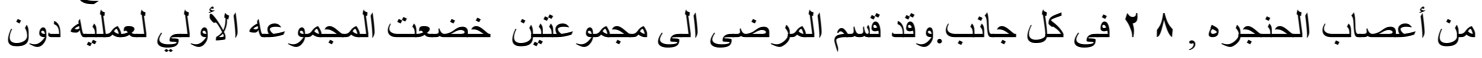
استخدام جهاز منبه العصب وخضعت المجمو عه الثانيه لعمليه باستخدام جهاز منبه العصب ثم مقارنه النتائج بين

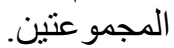

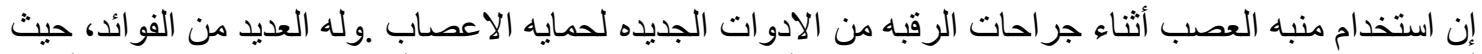

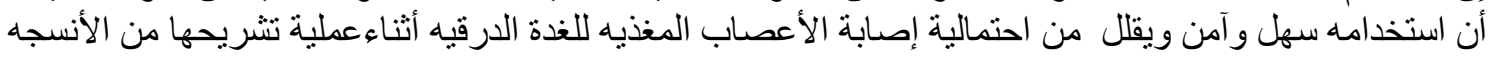

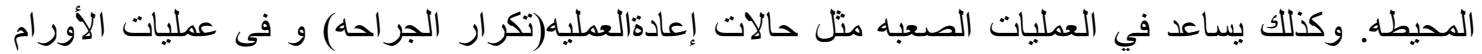

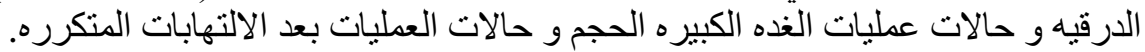

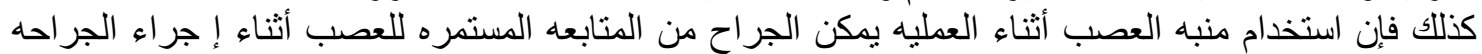

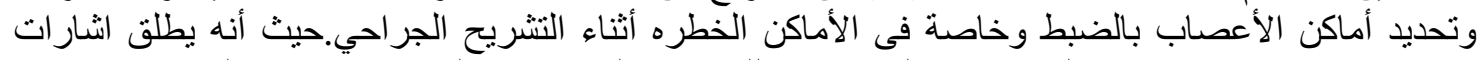

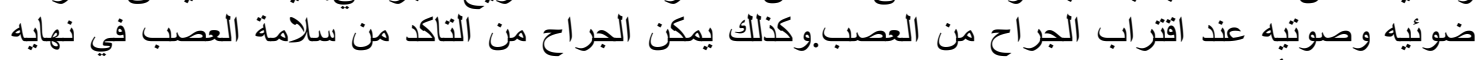
الجر احه.ويفيد أيضا في توجيه الجر احين المبندئين وتعليمهم مبادئ التشريح الجر احئ من الجئ

وبالرغم من هذه المزايا الكثيره للجهاز، قد لوحظ تقارب شديد فى النتائج بين المجموعه التي استخدمت منبه

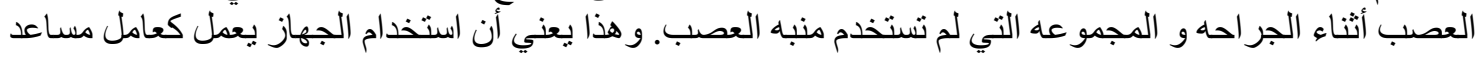

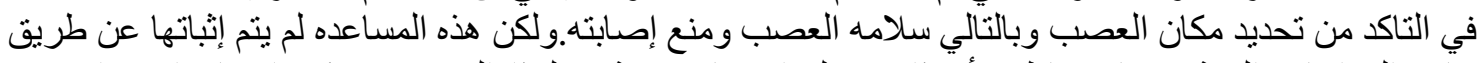

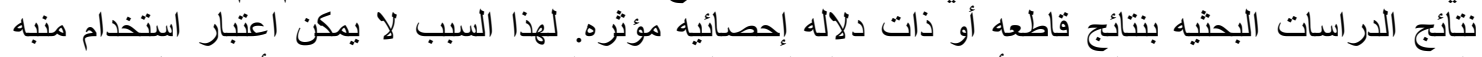

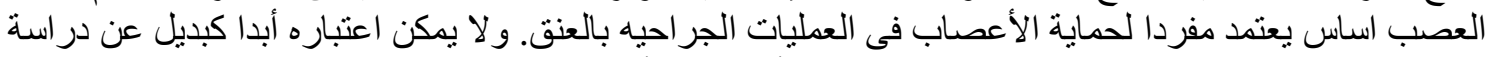

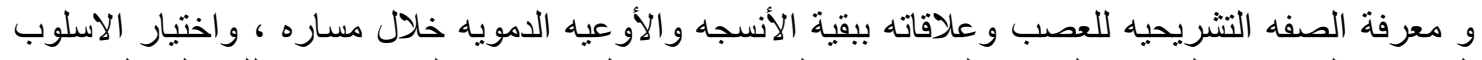

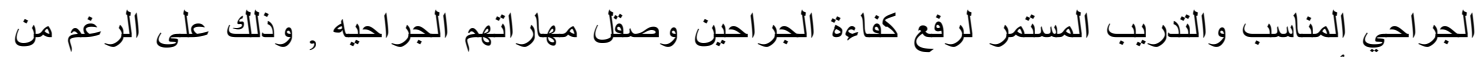
مساعدة الأجهزه لتحديد مكان العصب ولنب العمايته. 